\title{
Using Plant Functional Groups as a Strategy for Modeling Carbon Dynamics in Grassland Ecosystems
}

\author{
Deirdre Rogers ${ }^{1 *}$, Bailey Setzler ${ }^{1}$ and Yi-Wen Chiu ${ }^{2}$ \\ ${ }^{1}$ Research Assistant, Natural Resources Management and Environmental Sciences, California Polytechnic State \\ University, USA \\ ${ }^{2}$ Assistant Professor, Natural Resources Management and Environmental Sciences, California Polytechnic State \\ University, USA
}

\begin{abstract}
This paper argues for the use of plant functional groups as an important strategy for modeling carbon dynamics in grasslands. Carbon sequestration is paramount to help reduce climate change globally, and grasslands, representing $40 \%$ of all terrestrial area, can serve as primary locations of sequestration if optimal management strategies can be realized. Currently, the majority of research occurs in the field, which can take years to find what an optimal carbon sequestration strategy looks like, but modeling ecosystems offers an opportunity of realize long term management strategies for a particular locations. Current ecosystem modeling strategies tend to focus on agricultural applications or are model grassland for large regions, like Europe, or globally. Models tend to use just a few species, or only C3/C4 groups, as opposed to using C3 and C4 grasses as separate groups as well as forbs and legumes in their model. This paper lays out a justification for using the four groups in modeling in order to optimize simulation models while accounting for the importance of diversity in modeling strategies.
\end{abstract}

\section{Keywords}

Plant functional groups, Simulation models, Carbon sequestration

\section{Introduction}

The amount of carbon in the atmosphere has reached critical levels and, due to the greenhouse effect, the earth is predicted to warm by at least $1.5^{\circ} \mathrm{C}$ during the next century. The negative effects of climate change are increasingly evident, and the most recent IPCC report has estimated that we have 12-15 years to make drastic changes in atmospheric greenhouse gases to slow this trend [1]. If there is not a dramatic reduction in the amount of carbon, a $2{ }^{\circ} \mathrm{C}$ increase in global temperatures will be irreversible and the impacts of climate change including food insecurity, extreme weather events, rising sea levels, and more will be detrimental [1]. While there are a variety of potential methods of removing carbon from the atmosphere, manmade technologies that might sequester carbon are either not viable for large scale uptakes or are still in their infancy [2]. The most viable methods of carbon sequestration (CS) are through natural systems that remove carbon from the atmosphere and sequester it in terrestrial carbon pools. While there are numerous proposed pathways to achieve this goal, many of those systems are already taxed and will be difficult to restore within the short time period remaining; however, one promising terrestrial carbon sink is in grassland ecosystems.

Grasslands are one of the most viable options for CS be- cause they make up around $40 \%$ of the earth's surface [3] and account for $34 \%$ of total terrestrial carbon storage, with the vast majority (89\%) stored belowground in the soil [4]. In addition to managed natural grasslands, there are 1430 million hectares of abandoned agricultural grasslands globally that have experienced carbon losses due to poor management practices. It would take hundreds of years in order to mitigate the excess amount of atmospheric carbon from human emissions, carbon sequestration through purely natural processes in these abandoned lands [2], however, prior research has demonstrated that properly managing grasslands speed [2]. The rate of CS in grasslands is highly correlated to plant growth rate, the plant type, and species diversity [5]; and understanding the mechanisms of how ecosystem factors interact is key to determining successful management practices

*Corresponding author: Deirdre Sommerlad-Rogers, Research Assistant, Natural Resources Management and Environmental Sciences, California, USA, Tel: 509-339-3333

Accepted: May 22, 2019

Published online: May 24, 2019

Citation: Rogers D, Setzler B, Yi-Wen C (2019) Using Plant Functional Groups as a Strategy for Modeling Carbon Dynamics in Grassland Ecosystems. Adv Environ Stud 3(1):191-197 
under various climatic conditions. This proper management, which can include grazing, controlled burns, fertilization, liming, and many other strategies, could be the key to reducing atmospheric carbon levels and reversing the current trend of climate change.

In order to estimate the amount of carbon currently stored in grasslands and to identify long-term management strategies to increase CS, researchers have begun developing grassland models to simulate ecosystem processes. This requires modeling biotic and abiotic relationships in the ecosystem including plant-specific responses to and effects on the surrounding environment that are directly related to the rate in which an ecosystem sequesters atmospheric carbon. The challenges include understanding long term ecosystem dynamics in relation to CS. Simulation modeling allows researchers and land managers to plan and test management strategies that can potentially maximize sequestration on a longer time scale that would be more representative of ecosystem processes than empirical studies lasting 1-5 years. Research has shown that, over time, the interactions of plant communities change, which impacts how CS occurs [2]. This is especially important as the dominant grass species shift from $\mathrm{C} 3$ to $\mathrm{C} 4$ as plant communities mature, changing the rate at which carbon is sequestered. Because of the time it takes to engage in experimental research and the need for more immediate solutions, simulations become a critical tool to test ideal management strategies to maximize soil carbon. Kiplin, et al. [6] pointed out one of the main strategies that modeling simulations need to incorporate is the modeling of plant functional groups (FG). This paper discusses the current state of plant FG research in grasslands and examines how functional grouping can be used in simulation modeling.

\section{Carbon Sequestration in Grasslands}

The increase in atmospheric carbon levels is due in large part to emissions from terrestrial carbon pools. According to Lal [7], an estimated $136 \pm 55 \mathrm{Gt}$ of carbon was lost from the global terrestrial ecosystems from 1850-1998 caused by a variety of factors including soil degradation, erosion, deforestation, desertification and mineralization. While there are numerous ecologies that are effective in CS, grasslands present a widespread and fairly well studied ecosystem that has the potential to remove carbon from the atmospheric pool when certain management strategies are applied. According to Wu, et al. [8] 'Globally, grasslands store more than $10 \%$ of terrestrial biomass $C$ and $10-30 \%$ of soil organic $C(S O C)$, and they have been reported to sequester $C$ in soil at a rate of $0.5 \mathrm{Pg} \mathrm{C}$ year, which is about one-fourth of the potential $C$ sequestration in the world soils. 'There are many factors that influence the amount of carbon stored in ecosystems including climatic conditions and management practices such as grazing intensity and fertilizer additions [4]. These factors change plant community productivity, biomass allocation, nutrient availability, and microbial respiration rates that effectively change the carbon dynamics in grassland ecosystems.

In order to maximize the amount of carbon stored in terrestrial ecosystems, research continues to examine ecosys- tem dynamics [9] and the effects of different management strategies on grassland community health and long-term CS rates. The majority of research on grassland management effects on CS rates has been experimental field research, often with short time frames (6 months to 5 years), which has dramatically increased our understanding of grassland ecosystem dynamics, but there remains a limited amount of research on long terms CS strategies. This is unfortunate because it takes much longer for management activities to fully alter ecosystem processes, which has only recently been realized. In Yonekura, et al. [10] after new management practices were applied, simulations estimated that it would take about $30-50$ years before $C$ stocks reached equilibrium, much longer than the time frame of current field studies that seek to understand the effect of management on SOC stocks. The lack of long-term studies is partially because of the amount of time it takes to garner accurate results as well as difficulty establishing viable long-term empirical sites. In the absence of empirical data, scientists have turned to the use of cost-effective and timely simulation models as an alternative approach to understanding ecosystem carbon dynamics in response to various ecological and environmental driving forces. Despite the increase in adopting modeling approaches to aid in CS research, most studies fall short when attempting to incorporate the effects of community diversity into ecosystem simulations, creating potential deficits in modeling performance $[6,11]$.

Prior research has found that of the many factors that influence CS in terrestrial ecosystems, species diversity in grassland communities plays a significant role in CS optimization through creating vibrant eco-communities and more resilient ecosystems [11]. Eisenhauer, et al. [12] asserted that species diversity is critical in understanding the interactions between plants and soil, including ecosystem tolerance and biological adaptation to environmental stressors such as drought. For instance, some grass species can respond to higher temperatures by changing the length and depth of their roots allowing more effective use of below ground resources; and similar biological adaptation can occur above ground as well by altering leaf size and plant density to regulate soil temperature, with which can adversely impact belowground biomass and therefore CS rates.

Even though species diversity has been identified as a major factor affecting CS, many grassland models have focused on a single species or very simple mixes, undermining the ability of the model to characterize multi-species interactions that undoubtedly impact ecosystem functions such as productivity, nutrient cycling, and total CS [6]. Other simulations oversimplify modeling by including only phenology (percentage of plants that utilize C3 and C4 photosynthetic pathways), without indicating plant family classifications such as grasses, legumes, and for in their model $[8,13]$. For example, Wu, et al. [8] employed a complicated simulation model of the asymmetric productivity under altered precipitation over a 6-year period of time and stated that accounting for $\mathrm{C} 3$ and $\mathrm{C} 4$ grasses in their model limited the capacity of the proposed model. Using individual species remains a modeling challenge due to the overwhelming number of interactions between individ- 
uals in the community, especially for large ecosystems with high biodiversity. The use of functional groups in ecosystem simulations can overcome this hurdle by accounting for complimentary effects and species diversity, without the need to create an overly complex species-driven syntax.

\section{Functional Grouping and Traits}

Functional groups are defined by family classification: shrubs, forbs, legumes or grasses; and phenology: C3 or C4 photosynthetic pathway; both factors that give rise to distinctive functional traits among species [13]. C3, C4, forb, and legume FGs have been identified in various grasslands around the world [13-17]. These FGs have been shown to impact composition, succession, nitrogen $(\mathrm{N})$ dynamics and productivity in plant communities as well as CS in soils [14]. As Piao, et al. [5] pointed out, there is increasing indication that it is vegetation productivity that is driving carbon uptake, as opposed to other factors, so ensuring that species diversity is accounted for in simulation models is critical to ensuring model accuracy [15].

Natural grasslands are inhabited by an average of 8-20 plant species, whereas certain regions, such as the Eastern Tibetan Plateau, can have as many as 50 species per $\mathrm{m}^{2}$ [16]. Due to the increasing complexity resulting from the increase of plant species, many experiments intentionally limit the species richness in a study area, which can misrepresent the relationship between species diversity and CS, especially over time. Functional groupings are a potential way to simplify the modeling process by accounting for a variety of intraplant factors without the need to include the specific details about each species in the community. Functional group-based modeling approaches can simulate the plant-environment interaction by categorizing various biogeochemical mechanisms and pathways, which can improve computation efficiency by reducing algorithms. Understanding the unique qualities of FGs is important for considering why they might be used in simulation models.

\section{Grasses}

According to Munn [17] C3 and C4 grasses have very different responses to temperature, atmospheric carbon concentration, grazing intensity, and many other environmental factors. These differing responses can affect the FG's overall productivity and consequentially, CS. According to Abdalla, et al. [3], impacts of grazing intensity on SOC levels were significantly affected by grass type with $\mathrm{SOC}$ levels increasing under C4 grasses but decreasing under C3 grasses.

C3 grasses utilize the most common and simplistic photosynthetic pathway. They perform the Calvin Cycle by using the Rubisco enzyme to fix $\mathrm{CO}_{2}$, resulting in a $3-\mathrm{C}$ molecule as a product of photosynthesis. These grasses can thrive with higher atmospheric $\mathrm{CO}_{2}$ levels [18], tend to grow in cooler seasons and have lower temperature but higher moisture requirements than $\mathrm{C} 4$ grasses. $\mathrm{C} 3$ grasses tend to be fast growing with fine roots that have higher decomposition rates than plants with C4 photosynthetic pathways which can lead to greater CS rates among C3 rather than C4 grasses [18]. Research has shown that $\mathrm{C} 3$ grasses commonly dominate grass- land communities in the first 10 years but are eventually outcompeted by C4 grasses [2]. This is something that should be accounted for in modeling simulations.

C4 grasses are those that utilize a more complex photosynthetic mechanism that produces a 4-carbon molecule as a product of photosynthesis. Sixty percent of $\mathrm{C} 4$ species are found in the grasses, indicating their significance in determining long term grassland management strategies and modeling [19]. These plants concentrate $\mathrm{CO}_{2}$ inside of their stomata increasing the likelihood that $\mathrm{CO}_{2}$ binds to the Rubisco enzyme. This can increase their rate of photosynthesis, thereby increasing overall plant productivity as compared to C3 grasses [19]. C4 grasses also appears to have higher Nuptake efficiency, even though leaf $\mathrm{N}$ concentrations can be similar between $\mathrm{C} 3$ and C4 plants [19]. These plants have an adaptive advantage that leads them to respond very differently to environmental factors compared to other FGs. For example, Yang, et al. [2] found that $\mathrm{C} 4$ grasses had the greatest root mass which had the lowest decomposition rates. The high $\mathrm{N}$ use efficiency of $\mathrm{C} 4$ grass results in a relatively smaller investment of $\mathrm{N}$ in photosynthetic carboxylation enzymes, which impacts their growth [20]. Schneckenberger and Kuzyakov [21], in a study of Miscanthus $x$ giganteus, found that, when compared to maize (a C3 grass), C4 plants had significantly more SOC formation ( $0.23 \mathrm{~g}$ C4-C ( $\mathrm{kg}$ soil)-1y-1in loamy soil and $0.11 \mathrm{~g}$ C4-C (kg soil)$1 y$-1in the sandy soil), even after 12 years of maturity.

\section{Legumes}

The number of legumes present in grassland communities is smaller than that of grasses and forbs, however, they are very important for ecosystem functioning because legumes form a symbiotic relationship with $\mathrm{N}$ fixing bacteria. This bacteria lives in the roots of legumes and works to convert atmospheric $\mathrm{N}$ into a form available to plants, causing plants in legume FG to have their own unique interactions with ecosystem factors. Because legumes have an $\mathrm{N}$ fixing mechanism, they are unlikely to respond to $\mathrm{N}$ fertilization treatments and, on average, displayed $24 \%$ lower photosynthetic rate with $\mathrm{N}$ fertilization than the other FGs. This is drastically different than legumes because when no $\mathrm{N}$ is applied, legumes showed one of the highest rates of photosynthesis in comparison to other FGs. De Deyn, et al. [22] found that the presence of legumes in long term restoration practice significantly increase CS by $317 \mathrm{~g} \mathrm{C}$ and $35 \mathrm{~g} \mathrm{~N} \mathrm{~m} \sim 2$ year-1. The lack of response to $\mathrm{N}$ fertilization means that legumes are unlikely to increase their productivity and therefore will not contribute to and increase in CS rates in response to fertilization treatments compared to other FGs. Due to their unique $\mathrm{N}$ fixing bacteria, the presence of legumes in grassland ecosystems reduces soil $\mathrm{C} / \mathrm{N}$ ratios which stimulates microbial activity and leads to an overall increase in stored C in soils [23]. De Deyn, et al. [22] found that the presence of legumes in long term restoration practices significantly increase CS rates by $317 \mathrm{~g} \mathrm{C}$. Due to the significant change in CS rates caused by the presence of legumes, the differences between FGs must be accounted for in grassland modeling efforts.

\section{Forbs}

Forbs are broad-leafed herbaceous (non-woody) plants 
that differ from legumes in that they do not have a symbiotic relationship with $\mathrm{N}$ fixing bacteria and differ from grasses in that they are dicots whereas grasses are classified as monocots. Because these plants do not have a readily available source of $\mathrm{N}$, they are very sensitive to the addition of $\mathrm{N}$ to the system [14]. Forbs are essential to community composition and ecosystem functioning, especially for $C$ uptake and storage [16]. In one study of a restored tall grass prairie at Golden Moore Park in Illinois, grassland communities dominated by forbs or consisting of forb-grass mixes showed increased SOC levels, while grass-dominated communities experienced losses in total SOC levels [24]. Simulation models should account for the differences between forbs and grasses, rather than just photosynthetic pathway type because these functional groups have specific and different impacts on CS rates in grassland ecosystems.

\section{Functional Groups and the Complementary Effects}

Species diversity is linked to both productivity and CS in grasslands and should be accounted for in an accurate model of carbon dynamics. A growing amount of research has postulated the impact of diversity occurs belowground and is especially affected by differences in root distribution [25]. Due to the impact of below ground characteristics on productivity and resource use, it is important for land management strategies that maximize CS. Complementarity is the way in which different species interact including resource use, how they benefit other species, and their biotic feedbacks, resulting in complementary effects (CE) [26]. Species diversity accounts for a large part of the CE, which is how plants interact with each other for resources, leading to plants finding unique niches to exploit including deeper root depth and more diverse plant cover, helping to create more resilient communities with high productivity $[26,27]$. Trying to model each of these effects for each species within a grassland would create an unwieldly model, but FGsare a means of capturing how species compete and collaborate within an environment without adding to model complexity.

Use of functional traits in modeling may be able to account for interspecies interactions, especially if FGs are able to include both above and belowground traits as part of their grouping (specifically how group traits are selected and how these are scaled to community level effects, [15]) which can help model CE [27], and CS. They can also be used to account for both invasive and native species (each might be grouped separately) and FGs can also be used to check how well a grassland will resist invasion [28]. Many previous studies that have attempted to research CE have been limited by their lack of use of FGs throughout the course of the study.

To fully realize the potential for grasslands to engage in CS saturation, the maturity of the grassland is important. Thomas Clark, Lehman and Tilman [29] found that FGs will colonize different parts of a space, increasing the use of resources in a particular environment, demonstrating FG complimentary effects. While DeBoeck, et al. [30] found CE effects occurred through increased aboveground effects of the legumes FG, warming suppressed productivity, which could be attributed to negative competition. Unfortunately, the field experiment only ran for three years, limiting long term understanding on how the FGs might change in response to environmental changes. Yang, et al. [2] Models need to consider the interaction between time and species diversity, as biomass tends to increase linearly, but root mass plateaued towards the end of Yang, et al.'s [2] 22 year. Modeling allows for experimentation with what factors will maximize CS in grasslands over time so these strategies can be implemented today.

\section{Considerations for Functional Groups in Carbon Sequestration Modeling}

Functional groups serve as a strategy for representing plant communities and their relationships with the biotic and abiotic environment, without the need to input data for each individual species. Functional groups into a model. FGs are differentiated by their characteristics and corresponding relationships with various environmental factors as previously described. Wilson, et al. [31] concluded that phenology, biomass allocation, plant height, interception, and rooting depth caused different FGs to have differing impacts on evaporation, soil water availability, and drought conditions. The same concept is true for FGs and corresponding CS rates and Reich, et al. [14] confirms that differences in leaf area ratio, photosynthetic rate, and other characteristics between $\mathrm{C} 3$ grasses, C4 grasses, forbs, and legumes which resulted in differences in CS rate for these different FGs. These factors can be part of the simulation models to model community dynamics.

The distinction between C3 and C4 photosynthetic processes was recognized by Derner, et al. [32] as a factor that affects CS due to the FGs unique phenology. C3 and C4 groups should be incorporated into models to account for differing growth rates and photosynthetic $\mathrm{CO}_{2}$ uptake rate through FG leaf area index [33] to simulate productivity. Reich, et al. [14] also found the differences in leaf area ratio, photosynthetic rate, and other characteristics between C3 grasses, C4 grasses, forbs, and legumes which resulted in differences in CS rates for each FG. Following experimentation, Derner, et al. [32] concluded that overall, plants that utilize C4 photosynthetic processes contribute higher amounts of SOC. Specifically, greater than $80 \%$ of SOC in tall grass communities is associated with $\mathrm{C} 4$ relative to $\mathrm{C} 3$ plants and in short grass communities, $60-80 \%$ of SOC is derived from C4 species. This is supported by Acuna, Pastenes, and Villalobos [34] who compared SOC associated with turf grass species in Chile, demonstrating higher organic $\mathrm{C}$ content with $\mathrm{C} 4$ grasses than compared with C3 grasses. As such, long term models should consider how long it takes for the transition from C3 domination to C4 domination in modeling CS.

The abundance of forbs can also influence the amount of $C$ that is sequestered in the terrestrial ecosystem [24]. Ampleman, et al. [24] found the effect of community composition (C4 grass dominated vs. forb dominated) impacts SOC, with grass dominated lands losing carbon as compared to ones with more forbs. However, this study also points out that even though the overall SOC was increased under forb-dominated communities, forbs contributed to surface SOC stocks while C4 grasses impacting SOC stocks in 
deeper soil profiles. Depth of SOC stocks could be important for modeling long term carbon stocks, which are less likely to be disturbed at deeper levels than shallower ones. Due to the different impacts of forb and grass FGs on carbon cycling in ecosystems, their unique qualities must be accounted for in a comprehensive model.

Legumes and their unique characteristics must also be incorporated into a successful model. Canarini, et al. [23] studied drought effects on SOC in grasslands and discovered that even though legume biomass was much lower than the biomass of other FGs in the grassland, their presence resulted in a large increase in stored $\mathrm{C}$. This is hypothesized to be because the $\mathrm{N}$ fixed by the legumes symbiotic bacteria can also be used by plants of other FGs, increasing total available $\mathrm{N}$ and removing growth limits for other species [2]. Given that FGs differ significantly in these traits, these average values can be used in simulation modeling to represent species diversity effects on ecosystem functioning and ultimately, CS rates. Additionally, differentiating between forbs, legume, C3 and C4 grasses FGs is important for modeling grassland complexities.2019), allowing for increased community productivity and therefore increased CS rates throughout the ecosystem.

Both Rolinski, et al. [35] as well as Stöckle, Donatelli and Nelson [36] discussed dynamic vegetation modeling frameworks that has been tested, including the LPJmL, ORCHIDEE, as well as others. When thinking in terms of management strategies, creating FG based on functional traits of the groups would allow for greater nuances in the modeling. This is important when considering long terms CS modeling, as there is a need to figure out the potential for optimal and sustained CS, until soil saturation is reached. Simulation modeling using long term grassland species information would allow land managers to ensure that these plateaus are not reached prematurely, and may inform the use the management strategies including the incorporation of grazing, fire, and mowing strategies and allowing those to be included as part of long-term land management strategies and modeling factors. management strategies.

When modeling the characteristics of FGs, there are several key factors that should be included in order to properly simulate ecosystem processes. Biomass has a large impact on CS rates and can be integrated into models through data on net primary production [37] and vegetative growth as well as growth responses to management (grazing intensity, fertilizer addition, etc.), weather, and atmospheric C levels [38]. Growth is affected by photosynthetic rate which can be measured by LAI which currently exists for FGs and radiation use efficiency. (RUE) which can be calculated. Below ground factors should include vertical distribution of standing root biomass and root length density [39], leaf to root mass ratio [35], litter and root decomposition rates, as well as both long-term $\mathrm{N}$ and $\mathrm{C}$ strategies by group. Additionally, because since C3 and C4 plants have different growth responses to weather, inputs of seasonal responses by grouping would close some of the gaps in modeling that have been identified by Kipling, et al. [6].

Though some simulations have begun to model these key factors, much of this research focuses exclusively on agriculture. For example, Stockle, et al. [36] modeled crop yield by integrating $\mathrm{N}$ and $\mathrm{C}$ feedback loops, which can serve as a basic platform to quantify CS with proper modification. The work that has developed from this research could be tapped to give a baseline as to how to construct specific aspects of FG models and eventually apply them to models of CS in grassland ecosystems. Additionally, global models for C dynamics, such as that of Rolinski, et al. [35] can be scaled down to the local community level and integrate grassland management strategies into simulations to predict CS under specific ecosystem conditions and management strategies.

Not only would the use of FGs enhance the current models in simulating grassland ecosystem processes. During a study to identify strategies for improving models, Kipling, et al. [6] found that current models almost exclusively focus on the growing season and do not account for the effect of seasonal changes on plant communities. Because of this oversimplification, 'plant processes including hardening, dehardening and re-hardening, vernalisation, winter respiration, and allocation of carbohydrates to reserve tissues (which can all affect the status of the sward during the winter) are not sufficiently incorporated into most grassland models' causing estimates of grassland productivity to be less accurate than if these factors were accounted for. Use of FGs would significantly reduce the time required to add these factors to current models, making them more analogous to real-world conditions and improving scientific understanding of CS in grasslands.

\section{Conclusion}

In addition to reducing atmospheric $C$ levels, optimized management strategies that increase CS can also provide a multitude of benefits, including elevated grazing capacity, soil structure stability, increased biodiversity, improved water quality, resulting in improved ecosystem health $[7,36,40]$. Simulation of ecosystems can allow for managers and researchers to find strategies to increase grassland ecosystem sustainability and longevity without needing to wait 20-30 years to find out if their strategies work. Although the use of FGs in modeling technologies is still being validated by ongoing empirical studies, it is clear from prior research that plant traits are similar enough between FGs to be used in the representation of ecosystems in simulations. Use of FGs would streamline the modeling process and provide relevant data that would allow for the informed implementation of effective grassland management strategies to stimulate CS rates that would reduce atmospheric carbon levels.

\section{Acknowledgement}

This work is supported by Mclntire-Stennis, CALY1801, project accession no. 1013384 from the USDA National Institute of Food and Agriculture.

\section{References}

1. Intergovernmental Panel on Climate Change (IPCC) (2018) Global warming of $1.5^{\circ} \mathrm{C}$.

2. Yang $Y$, Tilman D, Furey G, et al. (2019) Soil carbon sequestration 
accelerated by restoration of grassland biodiversity. Nature Communications 10 .

3. Abdalla M, Hastings A, Chadwick DR, et al. (2018) Critical review of the impacts of grazing intensity on soil organic carbon storage and other soil quality indicators in extensively managed grasslands. Agric Ecosyst Environ 253: 62-81.

4. Eze S, Palmer SM, Chapman PJ (2018) Soil organic carbon stock and fractional distribution in upland grasslands. Geoderma 314: 175-183.

5. Piao S, Tan K, Nan H, et al. (2012) Impacts of climate and $\mathrm{CO}_{2}$ changes on the vegetation growth and carbon balance of Qinghai-Tibetan grasslands over the past five decades. Global and Planetary Change 98: 73-80.

6. Kipling RP, Virkajarvi P, Breitsameter L, et al. (2016) Key challenges and priorities for modelling European grasslands under climate change. Sci Total Environ. 566: 851-864.

7. Lal R (2004) Soil carbon sequestration to mitigate climate change. Geoderma 123: 1-22.

8. Wu D, Ciais P, Viovy N, et al. (2018) Asymmetric responses of primary productivity to altered precipitation simulated by ecosystem models across three long-term grassland sites. Biogeosciences 15: 3421-3437.

9. Arndal MF, Tolver A, Larsen KS, et al. (2018) Fine root growth and vertical distribution in response to elevated $\mathrm{co} 2$, warming and drought in a mixed heathland-grassland. Ecosystem 21: 15-30.

10. Yonekura $Y$, Ohta S, Kiyono $Y$, et al. (2012) Dynamics of soil carbon following destruction of tropical rainforest and the subsequent establishment of Imperata grassland in Indonesian Borneo using stable carbon isotopes. Global Change Biology 18: 2606-2616.

11. Fornara DA, TilmanD (2008) Plant functional composition influences rates of soil carbon and nitrogen accumulation. Journal of Ecology 96: 314-322.

12. Eisenhauer N, Hines J, Isbell F, et al. (2018) Plant diversity maintains multiple soil functions in future environments. ELife 7.

13. Hou Y, Zhou G, Xu Z, et al. (2013) Interactive effects of warming and increased precipitation on community structure and composition in an annual forb dominated desert steppe. PLoS ONE 8: e70114.

14. Reich PB, Buschena C, Tjoelker MG, et al. (2003) Variation in growth rate and ecophysiology among 34 grassland and savanna species under contrasting $\mathrm{N}$ supply: A test of functional group differences. New Phytologist 157: 617-631.

15. Funk JL, Larson JE, Ames GM, et al. (2017) Revisiting the holy grail: Using plant functional traits to understand ecological processes: Plant functional traits. Biological Reviews 92: $1156-1173$.

16. Li JH, Zhang J, Li WJ, et al. (2016) Plant functional groups, grasses versus forbs, differ in their impact on soil carbon dynamics with nitrogen fertilization. European Journal of Soil Biology 75: 79-87.

17. Munn RE (2002) Encyclopedia of global environmental change. Wiley, Chichester, New York.

18. Fornara DA, Tilman D (2012) Soil carbon sequestration in prairie grasslands increased by chronic nitrogen addition. Ecology 93: 2030-2036.

19. Taylor SH, Hulme SP, Rees M, et al. (2010) Ecophysiological traits in $\mathrm{C} 3$ and $\mathrm{C} 4$ grasses: A phylogenetically controlled screening experiment. New Phytologist 185: 780-791.
20. Wolf J, Ziska $L$ (2018) Comment on "Unexpected reversal of $C_{3}$ versus $\mathrm{C}_{4}$ grass response to elevated $\mathrm{CO}_{2}$ during a 20 -year field experiment". Science 361.

21. Schneckenberger K, Kuzyakov Y (2007) Carbon sequestration under Miscanthus in sandy and loamy soils estimated by natural ${ }^{13} \mathrm{C}$ abundance. Journal of Plant Nutrition and Soil Science 170: 538-542.

22. De Deyn GB, Shiel RS, Ostle NJ, et al. (2011) Additional carbon sequestration benefits of grassland diversity restoration: Soil $C$ sequestration and diversity restoration. Journal of Applied Ecology 48: 600-608.

23. Canarini A, Mariotte P, Ingram L, et al. (2018) Mineral-associated soil carbon is resistant to drought but sensitive to legumes and microbial biomass in an australian grassland. Ecosystems 21: 349-359.

24. Ampleman MD, Crawford KM, Fike DA (2014) Differential soil organic carbon storage at forb- and grass-dominated plant communities, 33 years after tallgrass prairie restoration. Plant and Soil 374: 899-913.

25. Mueller KE, Tilman D, Fornara DA, et al. (2013) Root depth distribution and the diversity-productivity relationship in a longterm grassland experiment. Ecology 94: 787-793.

26. Barry KE, Mommer L, van Ruijven J, et al. (2019) The Future of complementarity: Disentangling causes from consequences. Trends in Ecology \& Evolution 34: 167-180.

27. Zuo X, Zhang J, Lv P, et al. (2018) Effects of plant functional diversity induced by grazing and soil properties on aboveand belowground biomass in a semiarid grassland. Ecological Indicators 93: 555-561.

28. Byun C, de Blois S, Brisson J (2013) Plant functional group identity and diversity determine biotic resistance to invasion by an exotic grass. Journal of Ecology 101: 128-139.

29. Thomas Clark A, Lehman C, Tilman D (2018) Identifying mechanisms that structure ecological communities by snapping model parameters to empirically observed tradeoffs. Ecology Letters 21: 494-505.

30. De Boeck HD, Lemmens C, Nijs I (2008) Biomass production in experimental grasslands of different species richness during three years of climate warming. Biogeosciences 5: 585-594.

31. Wilson SD, Schlaepfer DR, Tietjen B (2018) Functional Group, Biomass, and Climate Change Effects on Ecological Drought in Semiarid Grasslands. JGR Biogeosciences 123: 1072-1085.

32. Derner JD, Boutton TW, Briske DD (2006) Grazing and ecosystem carbon storage in the north american great plains. Plant and Soil 280: 77-90.

33. Miguez FE, Maughan M, Bollero GA, et al. (2012) Modeling spatial and dynamic variation in growth, yield, and yield stability of the bioenergy crops Miscanthus $\times$ giganteus and Panicum virgatum across the conterminous United States. GCB Bioenergy 4: $509-520$.

34. Acuna E, Alejandra A, Pastenes V, et al. (2017) Carbon Sequestration and Photosynthesis in Newly Established Turfgrass Cover in Central Chile. Agronomy Journal 109: 397-405.

35. Rolinski S, Muller C, Heinke J, et al. (2018) Modeling vegetation and carbon dynamics of managed grasslands at the global scale with LPJmL 3.6. Geoscientific Model Development 11: 429-451.

36. Stockle CO, Donatelli M, Nelson R (2003) CropSyst, a cropping 
systems simulation model. European Journal of Agronomy 18 289-307.

37. Deng Q, Aras S, Yu CL, et al. (2017) Effects of precipitation changes on aboveground net primary production and soil respiration in a switchgrass field. Agriculture, Ecosystems \& Environment 248: 29-37.

38. Kawo S (2017) Estimating grass productivity under different clipping frequencies and rainfall amount: implications for rangeland responses to climate change (University of Hohenheim).
39. Siebenkas A, Roscher C (2016) Functional composition rather than species richness determines root characteristics of experimental grasslands grown at different light and nutrient availability. Plant and Soil 404: 399-412.

40. Causarano HJ, Doraiswamy PC, Muratova N, et al. (2011) Improved modeling of soil organic carbon in a semiarid region of Central East Kazakhstan using EPIC. Agronomy for Sustainable Development 31: 275-286. 